discriminate advanced (F3-F4) fibrosis was evaluated using AUROCs with 5-fold cross-validation repeated 100x. Optimal thresholds for F3-F4 fibrosis were selected based on the literature. Data presented are from an interim analysis on 1 May 2018.

Results A total of 4467 patients (median age 58 years, 55\% women, $72 \%$ Caucasian, 59\% with diabetes) were screened. In the 3220 with evaluable histology, median biopsy length was $2.0 \mathrm{~cm}, 8 \% \mathrm{~F} 0,9 \% \mathrm{~F} 1,13 \% \mathrm{~F} 2,31 \% \mathrm{~F} 3,40 \% \mathrm{~F} 4$, $59 \%$ with NAS $\geq 5$. Median values of NFS, FIB-4, ELF, and LS by TE increased with worsening fibrosis (-0.962/1.19/9.21/ $8.8 \mathrm{kPa}$ in $\mathrm{F} 0-\mathrm{F} 2$ vs $0.342 / 2.20 / 10.39 / 16.5 \mathrm{kPa}$ in $\mathrm{F} 3-\mathrm{F} 4$ respectively). AUROCs ranged from 0.75 to 0.80 to discriminate advanced fibrosis (table 1). When tests were combined, performance characteristics improved and PPVs $\geq 98 \%$ were possible.

Conclusions In these large, global phase 3 trials of SEL, routinely available NITs demonstrated acceptable diagnostic performance for the discrimination of advanced fibrosis due to NASH.

\section{IDDF2019-ABS-0134 SOFOSBUVIR/VELPATASVIR IS EFFECTIVE AND SAFE IN PATIENTS WITH CONCOMITANT PROTON PUMP INHIBITOR USE IN CLINICAL STUDIES}

\begin{abstract}
${ }^{1}$ Rafael Esteban*, ${ }^{2}$ Kosh Agarwal, ${ }^{3}$ Jose Luis Calleja, ${ }^{4}$ Jean-Francois Dufour, ${ }^{5}$ Steven Flamm, ${ }^{6}$ Stuart Gordon, ${ }^{7}$ Luisa Stamm, ${ }^{7}$ Liyun $\mathrm{Ni}^{7}{ }^{7}$ Diana Brainard, ${ }^{7}$ Christina Sze Man Yip, ${ }^{8}$ Kris Kowdley, ${ }^{9}$ Curtis Cooper, ${ }^{10}$ Juan Antonio Pineda, 'Lluis Castells, ${ }^{1}$ Maria Buti, ${ }^{11}$ Sabela Lens. 'Vall D'hebron University Hospital, Barcelona, Spain; ${ }^{2}$ King's College Hospital NHS Trust, London, UK; ${ }^{3}$ Hospital Universitario Puerta De Hierro, Madrid, Spain; ${ }^{4}$ University of Bern, Bern, Switzerland; ${ }^{5}$ Northwestern University Health System, Chicago, USA; ${ }^{6}$ Henry Ford Health System, Michigan, USA; ${ }^{7}$ Gilead Sciences, Inc., Foster City, USA; ${ }^{8}$ Swedish Medical Center, Seattle, Washington, USA; ${ }^{9}$ The Ottawa Hospital, Ottawa, Canada; ${ }^{10}$ Hospital Universitario Virgen De Valme, Sevilla, Spain; ${ }^{11}$ University of Barcelona, Barcelona, Spain
\end{abstract}

\subsection{6/gutjil-2019-IDDFabstracts.268}

Background Prior to the availability of Phase 1 drug interaction data, concomitant proton pump inhibitor (PPI) use was prohibited in clinical trials of sofosbuvir/velpatasvir (SOF/VEL). Later clinical studies allowed for the use of up to $20 \mathrm{mg}$ omeprazole or equivalent dosing. This analysis evaluated the efficacy and safety of patients with and without compensated cirrhosis who received SOF/VEL for 12 weeks and reported concomitant use of a PPI.

Methods This was a retrospective analysis from 12 Phase 2 and Phase 3 clinical studies in which patients of all genotypes with and without compensated cirrhosis received 12 weeks of SOF/VEL and reported concomitant use of a PPI. Efficacy was assessed by SVR12 and relapse rates and safety was assessed by treatment-emergent adverse events (AEs).

Results 87 patients reported concomitant use of a PPI. The mean age (range) was 57 years (26-78), 79\% were male and $75 \%$ white; $56 \%$ of patients were infected with genotype 3 and 29\% with genotype 1; 37\% of patients had compensated cirrhosis and $39 \%$ were treatment experienced. The most common PPI was omeprazole reported by $68 \%$ of patients.
The SVR12 rate was 97\% (84 of 87 patients). Of the 3 patients who did not achieve SVR12, 2 patients relapsed (relapse rate 2\%) and one patient with a history of diabetes discontinued SOF/VEL after 7 days of dosing due to hyperglycemia. No other patient had an AE which led to discontinuation or interruption of SOF/VEL. 78\% of patients had an AE, most of which were mild, and $11 \%$ had a serious AE. These efficacy and safety are comparable to patients enrolled in the same studies who received SOF/VEL for 12 weeks without concomitant use of a PPI (SVR12 rate 97\% [2445 of 2517 patients]; relapse rate 2\% [40 of 2488 patients]).

Conclusions In Phase 2 and Phase 3 clinical studies, the single-tablet regimen of SOF/VEL for 12 weeks was effective and safe in patients with concomitant PPI use. These data support the use of SOF/VEL according to labeled recommendations with respect to co-administration of PPIs and other acid reducing agents.

IDDF2019-ABS-0135
PHARMACOKINETICS OF ONCE-DAILY
SOFOSBUVIR OR LEDIPASVIR/SOFOSBUVIR
IN HCV-INFECTED PEDIATRICS AGED 3 TO

Rebecca Begley*, Amy Meng, Benedetta Massetto, Jiang Shao,Christina Sze Man Yip, John Ling, Anita Mathias. Gilead Sciences, Inc, Foster City, United States

\subsection{6/gutjnl-2019-IDDFabstracts.269}

Background SOF $400 \mathrm{mg}$ and LDV/SOF 90/400 $\mathrm{mg}$ are approved HCV treatment in adults and adolescents. Pharmacokinetic (PK) data indicates that SOF $200 \mathrm{mg}$ and LDV/SOF $45 / 200 \mathrm{mg}$ are appropriate doses in children 6 to $<12$ years old (y). SOF and LDV/SOF oral granules were developed; PK was assessed in children 3 to $<6 \mathrm{y}$.

Methods HCV-infected children (3 to $<6$ y) received SOF or LDV/SOF with doses selected to target exposures similar to adults: subjects $\geq 17 \mathrm{~kg}$ received SOF $200 \mathrm{mg}$ or LDV/SOF $45 / 200 \mathrm{mg}$; subjects <17 kg received SOF $150 \mathrm{mg}$ or LDV/ SOF $33.75 / 150 \mathrm{mg}$, QD. Subjects received SOF+RBV 12 or 24 weeks (GT2 or GT3, respectively) or LDV/SOF 12 weeks (GT1, 4). Intensive PK sampling (IPK lead-in) was performed on Day 7 (SOF; $\mathrm{N}=11$ ) or 10 (LDV/SOF; N=15). Exposures (AUCtau and Cmax) from subjects were compared to exposures in Phase 2/3 adult clinical programs. The primary endpoint was AUCtau of GS-331007 and LDV with predefined PK equivalence bounds of 50-200\%.

Results All but 1subject completed IPK assessments. At baseline, median age and weight for subjects was $5 \mathrm{y}$ and $17 \mathrm{~kg}$ in $\mathrm{SOF}+\mathrm{RBV} \mathrm{PK}$ lead-in $(\mathrm{N}=11)$ and $5 \mathrm{y}$ and $20 \mathrm{~kg}$ in LDV/SOF subjects $(\mathrm{N}=14)$. The predefined PK criteria were met as GS-331007 and LDV AUCtau were within the 50$200 \%$ boundaries as compared with the adult Phase 2/3 population (table 1). The GS-331007 Cmax (SOF+RBV) was modestly higher; these increases are not considered clinically relevant based on established exposure-safety analyses. SOF concentrations were within the range of those observed in the SOF and LDV/SOF adult Phase $2 / 3$ population (data not shown). 


\begin{tabular}{|c|c|c|c|}
\hline $\begin{array}{l}\text { PK parameters } \\
\text { Mean }(\% \mathrm{CV})\end{array}$ & $\begin{array}{l}3 \text { to }<6 \text { y } \\
\text { (Test) }\end{array}$ & $\begin{array}{l}\text { Ph2/3 Adult PopPK } \\
\text { (Reference) }^{b}\end{array}$ & $\begin{array}{l}\text { GLSM Ratio }(90 \% \text { Cl) } \\
\text { Test/Reference }\end{array}$ \\
\hline \multicolumn{4}{|c|}{ SOF + RBV ( $\mathrm{N}=10^{c} ;$ Test/Reference) } \\
\hline \multicolumn{4}{|l|}{ GS-331007 } \\
\hline$A U C_{\text {tau }}\left(h^{*} n g / m L\right)$ & $10300(18.1)$ & $7120(30.7)$ & $150(127,176)$ \\
\hline$C_{\max }(\mathrm{ng} / \mathrm{mL})$ & $1320(20.0)$ & $582(36.3)$ & $239(195,292)$ \\
\hline \multicolumn{4}{|c|}{ LDV/SOF ( $N=13^{d} ;$ Test/Reference) } \\
\hline \multicolumn{4}{|l|}{ LDV } \\
\hline$A U C_{\text {tau }}\left(h^{*} n g / m L\right)$ & $9300(34.6)$ & $8530(60.8)$ & $121(93.2,156)$ \\
\hline Cmax (ng/mL) & $531(28.5)$ & $364(51.4)$ & $158(126,197)$ \\
\hline \multicolumn{4}{|l|}{ GS-331007 } \\
\hline $\mathrm{AUC} C_{\mathrm{tau}}\left(\mathrm{hr}{ }^{*} \mathrm{ng} / \mathrm{mL}\right)$ & $11700(28.8)$ & $12500(29.2)$ & $94.0(82.5,107)$ \\
\hline$C_{\max }(\mathrm{ng} / \mathrm{mL})$ & $1000(23.3)$ & $736(28.2)$ & $138(121,157)$ \\
\hline
\end{tabular}

Conclusions SOF 200 or $150 \mathrm{mg}$ or LDV/SOF $45 / 200$ or $33.75 / 150 \mathrm{mg}$, for subjects $\geq 17 \mathrm{~kg}$ or $<17 \mathrm{~kg}$, respectively, were well tolerated and provided similar exposures to those observed in adults. These data support the ongoing evaluation of these doses in children 3 to $<6 \mathrm{y}$.

\section{IDDF2019-ABS-0136 SOFOSBUVIR/VELPATASVIR FOR 12 WEEKS IS SAFE AND EFFECTIVE IN PATIENTS UNDERGOING DIALYSIS}

\begin{abstract}
${ }^{1}$ Sergio Borgia*, ${ }^{2}$ Janet Dearden, ${ }^{3}$ Yoav Lurie, ${ }^{4}$ Stephen Shafran, ${ }^{5}$ Ashley Brown, ${ }^{6}$ Robert Hyland, ${ }^{6}$ Sophia Lu, ${ }^{6}$ Svetlana Markova, ${ }^{6}$ Hadas Dvory-Sobol, ${ }^{6}$ Anu Osinusi, ${ }^{6}$ Christina Sze Man Yip, ${ }^{7}$ Eric Yoshida, ${ }^{8}$ Jose Luis Calleja, ${ }^{9}$ Edward Gane, ${ }^{6}$ GS-US-342-4062 Investigator. 'William Osler Health System, ON, Canada; ${ }^{2}$ Saint Bartholomew's Hospital, UK; ${ }^{3}$ Shaare Zedek Medical Center, Israel; ${ }^{4}$ Department of Medicine, University of Alberta, AB, Canada; ${ }^{5}$ Imperial College Healthcare NHS Trust, UK; ${ }^{6}$ Gilead Sciences, Inc., Foster City, USA; ${ }^{7}$ Gordon and Leslie Diamond Health Care Centre, BC, Canada; ${ }^{8}$ Hospital Universitario Puerta De Hierro, Spain; ${ }^{9}$ Auckland City Hospital, New Zealand
\end{abstract}

\subsection{6/gutjnl-2019-IDDFabstracts.270}

Background Approved HCV treatments for patients on dialysis are associated with complexities including drug-drug interactions, baseline resistance testing and use of ribavirin. Despite higher concentrations of the primary circulating sofosbuvir (SOF) metabolite, GS-331007, in severe renal impairment, real-world cases demonstrated substantial use of SOF-based regimens in this population without safety concerns identified. This study evaluated the safety, efficacy, and pharmacokinetics (PK) of SOF/velpatasvir (VEL) for 12 weeks in patients with $\mathrm{HCV}$ infection on dialysis.

Methods Treatment-naive or -experienced patients, of any genotype, with or without compensated cirrhosis undergoing hemodialysis or peritoneal dialysis, were enrolled to receive open-label SOF/VEL (400/100 $\mathrm{mg})$ once daily for 12 weeks. The primary efficacy endpoint was a comparison of the SVR12 to a prespecified historic control rate of $83 \%$. The primary safety endpoint was the proportion of patients who discontinued therapy due to adverse events (AEs). Secondary endpoints included safety, viral resistance and PK.
Results 59 patients were enrolled at 21 sites in Canada, United Kingdom, Spain, Israel, Australia and New Zealand. The median age was 62 years (range 49-86), 59\% were male, 53\% white, 32\% treatment experienced, $29 \%$ had cirrhosis. Most patients had HCV genotype 1 (42\%), or 3 $(27 \%)$. Most (92\%) were on hemodialysis with a mean dialysis duration of 7.3 years. Treatment was well tolerated; no one discontinued therapy due to AEs. One patient was discontinued therapy on day 74 for non-compliance with $48 \%$ study medication adherence by pill count. Overall, 56/59 (95\%) of patients achieved SVR12. Two (3\%) had virologic relapse (one with non-adherence). One patient did not achieve SVR12 due to death from suicide after SVR4. Exposures were consistent with the Phase 1 renal impairment study. The most frequent AEs were headache, fatigue, nausea, and vomiting. Serious AEs occurred in $19 \%$ of patients, none was assessed as related to study drug.

Conclusions Treatment with SOF/VEL for 12 weeks in patients with and without cirrhosis undergoing dialysis resulted in a 95\% SVR12 rate. The regimen was safe and well-tolerated with no treatment-related discontinuations or treatment-related SAEs.

\section{IDDF2019-ABS-0138 CHANGES IN ORO-CECAL TRANSIT TIME (OCTT) AND PREVALENCE OF LACTOSE INTOLERANCE IN PATIENTS OF MICROSCOPIC COLITIS}

Balaji Bellam*, Siddharth Shukla, Rakesh Kochhar, Anupam Kumar Singh, Satyavathi Rana, Kaushal K Prasad, Sarthak Malik, Neha Berry, Megha Sharma, Saroj Kant Sinha. Post Graduate Institute of Medical Education and Research, Chandigarh, India

\subsection{6/gutjnl-2019-IDDFabstracts.271}

Background Microscopic colitis (MC) remains an elusive cause of chronic diarrhea. Histopathology is a gold standard with variable yield. The OCTT and concomitant lactose intolerance in these cases may aid to understand the complex symptoms. We used Hydrogen $(\mathrm{H} 2)$ breath tests $\left(\mathrm{H}_{2} \mathrm{BT}\right)$ for detecting SIBO (Small Intestinal Bacterial Overgrowth), Lactose intolerance and calculation of oro-cecal transit time (OCTT).

Methods 43 MC patients [mean age - 45.83( \pm 15.92 ] and 10 controls were studied. Among these, $37(86 \%)$ patients with MC \& 9 (90\%) controls underwent breath tests. SIBO was diagnosed with glucose $\mathrm{H}_{2} \mathrm{BT}$. For confirmation, we took rise $\geq 12$ ppm over fasting value in $\mathrm{H} 2$ concentration within 2hours of glucose ingestion Lactulose $\mathrm{H}_{2} \mathrm{BT}$ was done to calculate OCTT. Time taken for rising in breath hydrogen by $\geq 12 \mathrm{ppm}$ over baseline value in two consecutive readings was considered as OCTT. For lactose breath test, 15 minutes samples were taken up to 4 hours. A $\geq 20 \mathrm{ppm}$ rise over fasting value in $\mathrm{H}_{2}$ concentration in two consecutive readings was considered intolerance

Results Four (9.5\%) patients of SIBO in Glucose $\mathrm{H}_{2} \mathrm{BT}$ were enrolled in controls. Mean (+ SD) OCTT in cases of MC vs controls was $130.38 \pm 47.95 \mathrm{mins}$ and $97.14 \pm 48.55 \mathrm{mins}(\mathrm{p}=$ 0.109 ) respectively. Thus OCTT increased in $73 \%$ cases of MC vs 43\% controls. Also, in the MC group, 9(28.1\%) patients were lactose intolerant while vs 3(42.9\%) controls $(p=0.654)$. Thus a significant $28 \%$ MC patients were lactose intolerant possibly aggravating symptoms

Conclusions Lactose intolerance has lesser prevalence in cases of microscopic colitis. These patients were also detected to 